MSUHEP-50107

January, 1995

\title{
Effects of a heavy top quark on low energy data using the electroweak chiral Lagrangian
}

\author{
Ehab Malkawi and C.-P. Yuan \\ Department of Physics and Astronomy \\ Michigan State University \\ East Lansing, MI 48824
}

\begin{abstract}
We study large top quark mass effects on low energy physics in the chiral Lagrangian formulated electroweak theories. We show that these radiative corrections can be easily obtained from a set of Feynman diagrams involving only the scalar Goldstone bosons and the fermions when the contributions of the order $g$ (the weak coupling) are ignored. Using CERN LEP and SLAC Linear Collider (SLC) data we constrain on the nonstandard couplings, which might originate from the spontaneous symmetry-breaking sector, of the top quark to the electroweak gauge bosons.
\end{abstract}

PACS numbers: 14.65.Ha, 12.39.Fe, 12.60.-i 


\section{Introduction}

The use of the effective Lagrangian does not necessarily stem from our ignorance of the full dynamics. In fact, as pointed out by H. Georgi in Ref. [1], the effective field theory framework is not only simpler and more transparent, but it actually provides a deeper insight into the relevant physics. Commonly, the effective field theory approach is applied for estimating the size of higher order corrections [1]-[6]. In this paper we consider an effective field theory approach to electroweak radiative corrections and show how it can conveniently relate various radiative corrections important for testing the standard model (SM) in a rather elegant and clear way. More importantly, this approach is shown to clearly identify observables which are sensitive to the symmetrybreaking sector of the electroweak theories.

The strong evidence [7]- [10] for the presence of a heavy top quark motivates many studies on various scenarios in which new physics shows up in the top quark sector. For instance, in Ref. [11], we studied the general (nonstandard) couplings of the top quark to the electroweak (EW) gauge bosons in an effective chiral Lagrangian formulated electroweak theory with the spontaneously broken symmetry $\mathrm{SU}(2)_{L} \times$ $\mathrm{U}(1)_{Y} / \mathrm{U}(1)_{e m}$. The question regarding the origin of such nonstandard interactions is of a great importance, and was discussed to some extent in Ref. [11]. Here, we shall not repeat that discussion but to recall our conclusion that the previously announced LEP data [12] constrained somewhat the left-handed neutral current interaction. On the other hand, the right-handed neutral current and the charged currents were not usefully constrained by the data.

In this paper we will concentrate on two main points. The first point is to study the leading corrections of $O\left(m_{t}^{2} \ln \Lambda^{2}\right)$ to low energy observables arising from some nonstandard couplings of the top quark to the EW gauge bosons, parameterized in the chiral Lagrangian.] An earlier study was done in Ref. [11] by considering a gauge invariant set of Feynman diagrams in which massive gauge bosons appeared as external or internal lines. The Goldstone bosons and top quark only appeared as

\footnotetext{
${ }^{1} \Lambda$ is the cutoff scale at which the effective Lagrangian is valid.
} 
internal lines. Because the leading radiative corrections (in powers of $m_{t}$ ) are closely related to the spontaneous symmetry-breaking (SSB) sector, we expect that such corrections can be derived entirely from considering the interactions between the top quark and the scalar bosons (e.g., Goldstone bosons and possibly the Higgs boson). In this paper we develop a different formalism to calculate these leading corrections, using only the pure scalar boson and the top-bottom fermionic sectors in the chiral Lagrangian. We show how to reproduce the results obtained in Ref. [11] from a set of Feynman diagrams which only contain scalar boson and fermion lines.

The second point is to update the constraints on the nonstandard couplings of the top quark to the EW gauge bosons using the new LEP [13] and SLC data [14]. The rest of this paper is organized as follows. Sec. 2 is devoted to study the large top quark mass contribution (in powers of $m_{t}$ ) to low energy physics through the quantities $\rho$ and $\tau$ [15] in the chiral Lagrangian formulation. In Sec. 3 we update the constraints on the nonstandard couplings of the top quark to the EW gauge bosons. (Previous constraints were given in Ref. [11.) Sec. 4 contains some of our conclusions.

\section{$2 \quad$ Large $m_{t}$ effects to low energy physics}

In this paper we are interested in the chiral Lagrangian formulated electroweak theories in which the gauge symmetry $\mathrm{SU}(2)_{L} \times \mathrm{U}(1)_{Y}$ is nonlinearly realized. The relation between the linear and the nonlinear realization of $\mathrm{SU}(2)_{L} \times \mathrm{U}(1)_{Y}$ corresponds to some fixed nonlinear gauge transformation [16, 17]. The chiral Lagrangian approach has been used in understanding the low energy strong interactions because it can systematically describe the phenomena of spontaneous symmetry-breaking [18. Recently, the same technique has been widely used in studying the electroweak sector [2, 3, 4, 5, 19, 20, 21] to which this work has been directed.

A chiral Lagrangian can be constructed solely based upon the broken symmetry of the theory, and it is not necessary to specify the detailed dynamics of the actual breaking mechanism. Hence, it is the most general effective Lagrangian that can accommodate any underlying theory with that pattern of symmetry-breaking at the 
low energy scale. Furthermore, to study the low energy behavior of such a theory using the chiral Lagrangian approach, an expansion in powers of the external momentum is usually performed [22]. In this approach, one generally considers a Lie group $G$ which breaks down spontaneously into a subgroup $H$. For every broken generator a Goldstone boson is introduced in the effective theory [16]. In this paper we will concentrate on the electroweak group, $G=\mathrm{SU}(2)_{L} \times \mathrm{U}(1)_{Y}$ and $H=\mathrm{U}(1)_{e m}$. There are three Goldstone bosons, $\phi^{a}(a=1,2,3)$, generated by this breakdown, which are eventually eaten by $W^{ \pm}$and $Z$ gauge bosons and become their longitudinal degrees of freedom.

The Goldstone bosons transform nonlinearly under $G$ but linearly under the subgroup $H$. A convenient way to handle this is to introduce the matrix field

$$
\Sigma=\exp \left(i \frac{\phi^{a} \tau^{a}}{v_{a}}\right),
$$

where $\tau^{a}, a=1,2,3$ are the Pauli matrices normalized as $\operatorname{Tr}\left(\tau^{a} \tau^{b}\right)=2 \delta_{a b}$. The matrix field $\Sigma$ transforms under $G$ as

$$
\Sigma \rightarrow \Sigma^{\prime}=\exp \left(i \frac{\alpha^{a} \tau^{a}}{2}\right) \Sigma \exp \left(-i \frac{y \tau^{3}}{2}\right),
$$

where $\alpha^{1,2,3}$ and $y$ are the group parameters of $G$. Because of the $\mathrm{U}(1)_{e m}$ invariance, $v_{1}=v_{2}=v$, but they are not necessarily equal to $v_{3}$. In the $\mathrm{SM}, v(=246 \mathrm{GeV})$ is the vacuum expectation value of the Higgs boson field, and characterizes the scale of the symmetry-breaking. Also, $v_{3}=v$ arises from the approximate custodial symmetry present in the SM. It is this symmetry that is responsible for the tree-level relation

$$
\rho=\frac{M_{W}^{2}}{M_{Z}^{2} \cos ^{2} \theta_{W}}=1
$$

in the SM, where $\theta_{W}$ is the electroweak mixing angle, $M_{W}$ and $M_{Z}$ are the masses of $W^{ \pm}$and $Z$ boson, respectively. In this paper we assume the full theory guarantees that $v_{1}=v_{2}=v_{3}=v$.

It is convenient to define the composite fields

$$
\mathcal{W}_{\mu}^{a}=-i \operatorname{Tr}\left(\tau^{a} \Sigma^{\dagger} D_{\mu} \Sigma\right)
$$


and

$$
\mathcal{B}_{\mu}=g^{\prime} B_{\mu},
$$

where

$$
D_{\mu} \Sigma=\left(\partial_{\mu}-i g \frac{\tau^{a}}{2} W_{\mu}^{a}\right) \Sigma .
$$

In our notation $W_{\mu}^{a}$ and $B_{\mu}$ are the gauge bosons associated with the $\mathrm{SU}(2)_{L}$ and $\mathrm{U}(1)_{Y}$ groups, respectively. Also $g$ and $g^{\prime}$ are the corresponding gauge couplings. The composite fields transform under $G$ as

$$
\begin{gathered}
\mathcal{W}_{\mu}^{3} \rightarrow \mathcal{W}_{\mu}^{\prime 3}=\mathcal{W}_{\mu}^{3}-\partial_{\mu} y \\
\mathcal{W}_{\mu}^{ \pm} \rightarrow \mathcal{W}_{\mu}^{\prime \pm}=e^{ \pm i y} \mathcal{W}_{\mu}^{ \pm} \\
\mathcal{B}_{\mu} \rightarrow \mathcal{B}_{\mu}^{\prime}=\mathcal{B}_{\mu}+\partial_{\mu} y
\end{gathered}
$$

where

$$
\mathcal{W}_{\mu}^{ \pm}=\frac{\mathcal{W}_{\mu}^{1} \mp i \mathcal{W}_{\mu}^{2}}{\sqrt{2}} .
$$

We also introduce the composite fields $\mathcal{Z}_{\mu}$ and $\mathcal{A}_{\mu}$ as

$$
\begin{gathered}
\mathcal{Z}_{\mu}=\mathcal{W}_{\mu}^{3}+\mathcal{B}_{\mu}, \\
s^{2} \mathcal{A}_{\mu}=s^{2} \mathcal{W}_{\mu}^{3}-c^{2} \mathcal{B}_{\mu},
\end{gathered}
$$

where $s^{2} \equiv \sin ^{2} \theta_{W}$, and $c^{2}=1-s^{2}$. In the unitary gauge $(\Sigma=1)$

$$
\begin{aligned}
\mathcal{W}_{\mu}^{a} & =-g W_{\mu}^{a}, \\
\mathcal{Z}_{\mu} & =-\frac{g}{c} Z_{\mu}, \\
\mathcal{A}_{\mu} & =-\frac{e}{s^{2}} A_{\mu},
\end{aligned}
$$

where we have used the relations $e=g s=g^{\prime} c, W_{\mu}^{3}=c Z_{\mu}+s A_{\mu}$, and $B_{\mu}=-s Z_{\mu}+$ $c A_{\mu}$. The transformations of $\mathcal{Z}_{\mu}$ and $\mathcal{A}_{\mu}$ under $G$ are

$$
\begin{gathered}
\mathcal{Z}_{\mu} \rightarrow \mathcal{Z}_{\mu}^{\prime}=\mathcal{Z}_{\mu}, \\
\mathcal{A}_{\mu} \rightarrow \mathcal{A}_{\mu}^{\prime}=\mathcal{A}_{\mu}-\frac{1}{s^{2}} \partial_{\mu} y .
\end{gathered}
$$


Hence, under $G$ the fields $\mathcal{W}_{\mu}^{ \pm}$and $\mathcal{Z}_{\mu}$ transform as vector fields, but $\mathcal{A}_{\mu}$ transforms as a gauge boson field which plays the role of the photon field $A_{\mu}$.

Using the fields defined as above, one may construct the $\mathrm{SU}(2)_{L} \times \mathrm{U}(1)_{Y}$ gauge invariant interaction terms in the chiral Lagrangian

$$
\begin{aligned}
\mathcal{L}^{B}= & -\frac{1}{4 g^{2}} \mathcal{W}_{\mu \nu}^{a} \mathcal{W}^{a \mu \nu}-\frac{1}{4 g^{\prime 2}} \mathcal{B}_{\mu \nu} \mathcal{B}^{\mu \nu} \\
& +\frac{v^{2}}{4} \mathcal{W}_{\mu}^{+} \mathcal{W}^{-\mu}+\frac{v^{2}}{8} \mathcal{Z}_{\mu} \mathcal{Z}^{\mu}+\ldots
\end{aligned}
$$

where

$$
\begin{gathered}
\mathcal{W}_{\mu \nu}^{a}=\partial_{\mu} \mathcal{W}_{\nu}^{a}-\partial_{\nu} \mathcal{W}_{\mu}^{a}+\epsilon^{a b c} \mathcal{W}_{\mu}^{b} \mathcal{W}_{\nu}^{c} \\
\mathcal{B}_{\mu \nu}=\partial_{\mu} \mathcal{B}_{\nu}-\partial_{\nu} \mathcal{B}_{\mu}
\end{gathered}
$$

and where ... denotes other possible four- or higher- dimensional operators [2, 4].

It is easy to show that ${ }^{2}$

$$
\mathcal{W}_{\mu \nu}^{a} \tau^{a}=-g \Sigma^{\dagger} W_{\mu \nu}^{a} \tau^{a} \Sigma
$$

and

$$
\mathcal{W}_{\mu \nu}^{a} \mathcal{W}^{a \mu \nu}=g^{2} W_{\mu \nu}^{a} W^{a \mu \nu}
$$

This simply reflects the fact that the kinetic term is not related to the Goldstone bosons sector, i.e., it does not originate from the symmetry-breaking sector.

The mass terms in Eq. (18) can be expanded as

$$
\begin{aligned}
\frac{v^{2}}{4} \mathcal{W}_{\mu}^{+} \mathcal{W}^{-\mu}+\frac{v^{2}}{8} \mathcal{Z}_{\mu} \mathcal{Z}^{\mu}= & \partial_{\mu} \phi^{+} \partial^{\mu} \phi^{-}+\frac{1}{2} \partial_{\mu} \phi^{3} \partial^{\mu} \phi^{3} \\
& +\frac{g^{2} v^{2}}{4} W_{\mu}^{+} W^{\mu-}+\frac{g^{2} v^{2}}{8 c^{2}} Z_{\mu} Z^{\mu}+\ldots
\end{aligned}
$$

At the tree-level, the mass of $W^{ \pm}$boson is $M_{W}=g v / 2$ and the mass of $Z$ boson is $M_{Z}=g v / 2 c$. The above identity implies that the radiative corrections to the mass of the gauge bosons can be related to the wavefunction renormalization of the Goldstone bosons, cf. Eq. (42), and therefore sensitive to the symmetry-breaking sector.

\footnotetext{
${ }^{2}$ Use $\mathcal{W}_{\mu}^{a} \tau^{a}=-2 i \Sigma^{\dagger} D_{\mu} \Sigma$, and $\left[\tau^{a}, \tau^{b}\right]=2 i \epsilon^{a b c} \tau^{c}$.
} 
Fermions can be included in this context by assuming that each flavor transforms under $G=\mathrm{SU}(2)_{L} \times \mathrm{U}(1)_{Y}$ as 19

$$
f \rightarrow f^{\prime}=e^{i y Q_{f}} f
$$

where $Q_{f}$ is the electric charge of $f$.

Out of the fermion fields $f_{1}, f_{2}$ (two different flavors), and the Goldstone bosons matrix field $\Sigma$, the usual linearly realized fields $\Psi$ can be constructed. For example, the left-handed fermions $\left[\mathrm{SU}(2)_{L}\right.$ doublet] are

$$
\Psi_{L}=\Sigma F_{L}=\Sigma\left(\begin{array}{l}
f_{1} \\
f_{2}
\end{array}\right)_{L}
$$

with $Q_{f_{1}}-Q_{f_{2}}=1$. One can easily show that $\Psi_{L}$ transforms linearly under $G$ as

$$
\Psi_{L} \rightarrow \Psi_{L}^{\prime}=\mathrm{g} \Psi_{L}
$$

where $\mathrm{g}=\exp \left(i \frac{\alpha^{a} \tau^{a}}{2}\right) \exp \left(i \frac{y}{2}\right) \in G$. Linearly realized right-handed fermions $\Psi_{R}$ $\left[\mathrm{SU}(2)_{L}\right.$ singlet] simply coincide with $F_{R}$, i.e.,

$$
\Psi_{R}=F_{R}=\left(\begin{array}{l}
f_{1} \\
f_{2}
\end{array}\right)_{R} .
$$

It is then straightforward to construct a chiral Lagrangian containing both the bosonic and the fermionic fields defined as above .

Our goal is to study the large Yukawa corrections to the low energy data from the chiral Lagrangian formulated electroweak theories. We shall separate the radiative corrections as an expansion in both the Yukawa coupling $g_{t}$ and the weak coupling $g$. $\left(g_{t}=\sqrt{2} m_{t} / v\right.$, where $m_{t}$ is the mass of the top quark.) With this separation we can then consider the case where corrections of the order $g$ are ignored compared to those of $g_{t}$. This kind of study was done for the SM in Ref. [15], where the gauge bosons were considered as classical fields so that the full gauge invariance of the SM Lagrangian was maintained, and a set of Ward identities was derived to relate the Green's functions of the Goldstone bosons and the gauge bosons. Hence, large $g_{t}$ corrections can be easily obtained from calculating Feynman diagrams involving only fermions (top and bottom quarks) and scalar bosons (e.g., Goldstone bosons and 
possibly the Higgs boson) but not gauge bosons. The same conclusion can be drawn using the chiral Lagrangian approach in a far more elegant and clear way, as shown below in this section.

Why is the chiral Lagrangian formulation useful in finding large $g_{t}$ corrections beyond the tree-level? In general to perform a loop calculation, one needs to fix a gauge and therefore explicitly destroys the gauge invariance $\left[\mathrm{SU}(2)_{L} \times \mathrm{U}(1)_{Y}\right]$ of the Lagrangian. However, to find the large $g_{t}$ corrections one does not need to include gauge bosons in loops [15]. Thus, there is no need to fix a gauge and the full gauge invariance of the effective Lagrangian is maintained. Because the chiral Lagrangian possesses the $\mathrm{SU}(2)_{L} \times \mathrm{U}(1)_{Y}$ invariance (nonlinearly) and the $\mathrm{U}(1)_{\text {em }}$ invariance (linearly) at any given order of the perturbative expansions, and all the loop corrections can be reorganized using the composite fields $\mathcal{W}_{\mu}^{ \pm}, \mathcal{Z}_{\mu}$, and $\mathcal{A}_{\mu}$ in a gauge invariant form, therefore, it is the most convenient and elegant way to find $g_{t}$ corrections beyond the tree-level. This is obvious because the leading radiative corrections (in powers of $\left.m_{t}\right)$ are products of the SSB and therefore independent of the weak gauge coupling $g$. We note that in the expansion of the field

$$
\mathcal{Z}_{\mu}=\frac{2}{v} \partial_{\mu} \phi^{3}-\frac{g}{c} Z_{\mu}+\ldots
$$

there is always a factor $g$ associated with a weak gauge boson field. Hence, loop corrections independent of the gauge coupling $g$ can be obtained by simply considering the scalar and the fermionic sectors in the theory. In the following we shall show how this is done.

\subsection{Effective Lagrangian}

To obtain the large contributions of the top quark mass (in powers of $m_{t}$ ) to low energy data, we need only to concentrate on the top-bottom fermionic sector $\left(f_{1}=t\right.$ and $f_{2}=b$ ) in addition to the bosonic sector. The most general gauge invariant chiral Lagrangian can be written as

$$
\mathcal{L}_{0}=i \bar{t} \gamma^{\mu}\left(\partial_{\mu}+i \frac{2 s_{0}^{2}}{3} \mathcal{A}_{\mu}\right) t+i \bar{b} \gamma^{\mu}\left(\partial_{\mu}-i \frac{s_{0}^{2}}{3} \mathcal{A}_{\mu}\right) b
$$




$$
\begin{aligned}
& -\left(\frac{1}{2}-\frac{2 s_{0}^{2}}{3}+\kappa_{L}^{\mathrm{NC}}\right) \overline{t_{L}} \gamma^{\mu} t_{L} \mathcal{Z}_{\mu}-\left(\frac{-2 s_{0}^{2}}{3}+\kappa_{R}^{\mathrm{NC}}\right) \overline{t_{R}} \gamma^{\mu} t_{R} \mathcal{Z}_{\mu} \\
& -\left(\frac{-1}{2}+\frac{s_{0}^{2}}{3}\right) \overline{b_{L}} \gamma^{\mu} b_{L} \mathcal{Z}_{\mu}-\frac{s_{0}^{2}}{3} \overline{b_{R}} \gamma^{\mu} b_{R} \mathcal{Z}_{\mu} \\
& -\frac{1}{\sqrt{2}}\left(1+\kappa_{L}^{\mathrm{CC}}\right) \overline{t_{L}} \gamma^{\mu} b_{L} \mathcal{W}_{\mu}^{+}-\frac{1}{\sqrt{2}}\left(1+\kappa_{L}^{\mathrm{CC}}\right) \overline{b_{L}} \gamma^{\mu} t_{L} \mathcal{W}_{\mu}^{-} \\
& -\frac{1}{\sqrt{2}} \kappa_{R}^{\mathrm{CC}} \overline{t_{R}} \gamma^{\mu} b_{R} \mathcal{W}_{\mu}^{+}-\frac{1}{\sqrt{2}} \kappa_{R}^{\mathrm{CC}} \overline{b_{R}} \gamma^{\mu} t_{R} \mathcal{W}_{\mu}^{-} \\
& -m_{t} \bar{t} t+\ldots,
\end{aligned}
$$

where $\kappa_{L}^{\mathrm{NC}}, \kappa_{R}^{\mathrm{NC}}, \kappa_{L}^{\mathrm{CC}}$, and $\kappa_{R}^{\mathrm{CC}}$ parameterize possible deviations from the SM predictions [11], and ... indicates possible Higgs boson interactions and other higher dimensional operators. Here we have assumed that new physics from the SSB sector might modify the interactions of the top quark to the EW gauge bosons. On the other hand, the bare $b-b-Z$ couplings are not modified in the limit of ignoring the mass of the bottom quark [11]. The subscript 0 denotes bare quantities and all the fields in the Lagrangian $\mathcal{L}_{0}$, Eq. (29), are bare fields.

Needless to say, the composite fields are only used to organize the radiative corrections in the chiral Lagrangian. To actually calculate loop corrections one should expand these operators in terms of the Goldstone boson and the gauge boson fields. The gauge invariant result of loop calculations can be written in a form similar to Eq. (29). Denoting the fermionic part of this effective Lagrangian as $\mathcal{L}_{\text {eff }}$, then

$$
\begin{aligned}
\mathcal{L}_{\text {eff }}= & i Z_{b}^{L} \overline{b_{L}} \gamma_{\mu} \partial^{\mu} b_{L}+Z_{1} \frac{s_{0}^{2}}{3} \overline{b_{L}} \gamma_{\mu} b_{L} \mathcal{A}^{\mu}+\frac{1}{2}\left(Z_{v}^{L}-Z_{2} \frac{2 s_{0}^{2}}{3}\right) \overline{b_{L}} \gamma_{\mu} b_{L} \mathcal{Z}^{\mu} \\
& +i Z_{b}^{R} \overline{b_{R}} \gamma_{\mu} \partial^{\mu} b_{R}+Z_{3} \frac{s_{0}^{2}}{3} \overline{b_{R}} \gamma_{\mu} b_{R} \mathcal{A}^{\mu}-Z_{4} \frac{s_{0}^{2}}{3} \overline{b_{R}} \gamma_{\mu} b_{R} \mathcal{Z}^{\mu}+\ldots
\end{aligned}
$$

in which the coefficient functions $Z_{1}, Z_{2}, Z_{3}, Z_{4}, Z_{b}^{L}, Z_{b}^{R}$, and $Z_{v}^{L}$ contain all the loop corrections, and all the fields in $\mathcal{L}_{\text {eff }}$ are bare fields.

In the case of ignoring the corrections of the order $g$, the effective Lagrangian can be further separated into two parts: one part has the explicit linear $\mathrm{U}(1)_{Y}$ symmetry in the unitary gauge, and the other part contains all the radiative corrections which do not vanish when taking the $g \rightarrow 0$ limit. Specifically, in this approximation, we 
can write

$$
\begin{aligned}
\mathcal{L}_{\text {eff }}= & i Z_{b}^{L} \overline{b_{L}} \gamma_{\mu} \partial^{\mu} b_{L}-Z_{1} \frac{1}{3} \overline{b_{L}} \gamma_{\mu} b_{L} \mathcal{B}^{\mu}+\frac{1}{2} Z_{v}^{L} \overline{b_{L}} \gamma_{\mu} b_{L} \mathcal{Z}^{\mu} \\
& +i Z_{b}^{R} \overline{b_{R}} \gamma_{\mu} \partial^{\mu} b_{R}-Z_{3} \frac{1}{3} \overline{b_{R}} \gamma_{\mu} b_{R} \mathcal{B}^{\mu}+\ldots
\end{aligned}
$$

where

$$
\mathcal{B}_{\mu}=s_{0}^{2}\left(\mathcal{Z}_{\mu}-\mathcal{A}_{\mu}\right)
$$

derived from Eqs. (11) and (12). Note that as shown in Eqs. (5) and (9) the field $\mathcal{B}_{\mu}$ is not composite and transforms exactly like $B_{\mu}$. Comparing Eq. (30) with (31), we conclude that the coefficient functions $Z_{1}, Z_{2}, Z_{3}$, and $Z_{4}$ must be related and

$$
\begin{aligned}
& Z_{2}=Z_{1}, \\
& Z_{4}=Z_{3} .
\end{aligned}
$$

All the radiative corrections to the vertex $b-b-\phi^{3}$ in powers of $m_{t}$ are summarized by the coefficient function $Z_{v}^{L}$ because, from Eq. (28),

$$
\frac{1}{2} Z_{v}^{L} \overline{b_{L}} \gamma_{\mu} b_{L} \mathcal{Z}^{\mu}=Z_{v}^{L} \frac{1}{v} \overline{b_{L}} \gamma_{\mu} b_{L} \partial^{\mu} \phi^{3}+\ldots
$$

Since the effective Lagrangian $\mathcal{L}_{\text {eff }}$ possesses an explicit $\mathrm{U}(1)_{\text {em }}$ symmetry and under $G$ the field $\mathcal{A}_{\mu}$ transforms as a gauge boson field and $\mathcal{Z}_{\mu}$ as a neutral vector boson field, therefore, based upon the Ward identities in QED we conclude that in Eq. (30)

$$
Z_{1}=Z_{b}^{L}
$$

and

$$
Z_{3}=Z_{b}^{R}
$$

Hence, the effective Lagrangian $\mathcal{L}_{\text {eff }}$ can be rewritten as

$$
\begin{aligned}
\mathcal{L}_{e f f}= & i Z_{b}^{L} \overline{b_{L}} \gamma^{\mu}\left(\partial_{\mu}-i \frac{s_{0}^{2}}{3} \mathcal{A}_{\mu}\right) b_{L}+i Z_{b}^{R} \overline{b_{R}} \gamma^{\mu}\left(\partial_{\mu}-i \frac{s_{0}^{2}}{3} \mathcal{A}_{\mu}\right) b_{R} \\
& +\frac{1}{2}\left(Z_{v}^{L}-Z_{b}^{L} \frac{2 s_{0}^{2}}{3}\right) \overline{b_{L}} \gamma_{\mu} b_{L} \mathcal{Z}^{\mu}-Z_{b}^{R} \frac{s_{0}^{2}}{3} \overline{b_{R}} \gamma_{\mu} b_{R} \mathcal{Z}^{\mu}+\ldots
\end{aligned}
$$


This effective Lagrangian summarizes all the loop corrections in powers of $m_{t}$ in the coefficient functions $Z_{b}^{L}, Z_{b}^{R}$, and $Z_{v}^{L}$. Recall that up to now all the fields in $\mathcal{L}_{\text {eff }}$ are bare fields. To compare with the low energy data we prefer to express $\mathcal{L}_{\text {eff }}$ in terms of the renormalized fields. In Eq. (38), the kinetic terms of the $b_{L}$ and $b_{R}$ fields can be properly normalized after redefining (renormalizing) the fields $b_{L}$ and $b_{R}$ by $\left(Z_{b}^{L}\right)^{\frac{-1}{2}} b_{L}$ and $\left(Z_{b}^{R}\right)^{\frac{-1}{2}} b_{R}$, respectively. In terms of the renormalized fields $b_{L}$ and $b_{R}$, $\mathcal{L}_{\text {eff }}$ can be rewritten as

$$
\begin{aligned}
\mathcal{L}_{\text {eff }}= & \overline{b_{L}} i \gamma^{\mu}\left(\partial_{\mu}-i \frac{s_{0}^{2}}{3} \mathcal{A}_{\mu}\right) b_{L}+\overline{b_{R}} i \gamma^{\mu}\left(\partial_{\mu}-i \frac{s_{0}^{2}}{3} \mathcal{A}_{\mu}\right) b_{R} \\
& +\frac{1}{2}\left(\frac{Z_{v}^{L}}{Z_{b}^{L}}-\frac{2 s_{0}^{2}}{3}\right) \overline{b_{L}} \gamma_{\mu} b_{L} \mathcal{Z}^{\mu}-\frac{s_{0}^{2}}{3} \overline{b_{R}} \gamma_{\mu} b_{R} \mathcal{Z}^{\mu}+\ldots
\end{aligned}
$$

Before considering the physical observables at low energy let us first examine the bosonic sector. Similar to our previous discussions, loop corrections to the bosonic sector can be organized using the effective Lagrangian

$$
\begin{aligned}
\mathcal{L}_{\text {eff }}^{B}= & -\frac{1}{4 g_{0}^{2}} \mathcal{W}_{\mu \nu}^{a} \mathcal{W}^{\mu \nu a}-\frac{1}{4 g_{0}^{2}} \mathcal{B}_{\mu \nu} \mathcal{B}^{\mu \nu} \\
& +Z^{\phi} \frac{v_{0}^{2}}{4} \mathcal{W}_{\mu}^{+} \mathcal{W}^{-\mu}+Z^{\chi} \frac{v_{0}^{2}}{8} \mathcal{Z}_{\mu} \mathcal{Z}^{\mu}+\ldots
\end{aligned}
$$

Note that in the above equation we have explicitly used the subscript 0 to indicate bare quantities. The bosonic Lagrangian in Eq. (18) and the identity in Eq. (22) imply that the Yang-Mills terms (the first two terms in $\mathcal{L}^{B}$ ) are not directly related to the SSB sector. Hence, any radiative corrections to the field $W_{\mu \nu}^{a}$ must know about the weak coupling $g$, i.e., suppressed by $g$ in our point of view. This also holds for operators, of dimension four or higher, which include $W_{\mu \nu}^{a}$ in the chiral Lagrangian where all these gauge invariant terms are suppressed by the weak coupling $g$ [2, 4]. The same conclusion applies to $B_{\mu \nu}$. Therefore we conclude that the fields $\mathcal{W}_{\mu}^{ \pm}, \mathcal{Z}_{\mu}$, and $\mathcal{A}_{\mu}$ in $\mathcal{L}_{\text {eff }}$ and $\mathcal{L}_{\text {eff }}^{B}$ do not get wavefunction corrections (renormalization) in the limit of ignoring corrections of the order $g$, namely the renormalized fields and the bare fields are identical in this limit.

Expanding the mass terms in Eq. (40) we find

$$
Z^{\phi} \frac{v_{0}^{2}}{4} \mathcal{W}_{\mu}^{+} \mathcal{W}^{-\mu}+Z^{\chi} \frac{v_{0}^{2}}{8} \mathcal{Z}_{\mu} \mathcal{Z}^{\mu}=Z^{\phi} \partial_{\mu} \phi^{+} \partial^{\mu} \phi^{-}+\frac{1}{2} Z^{\chi} \partial_{\mu} \phi^{3} \partial^{\mu} \phi^{3}
$$




$$
+Z^{\phi} \frac{g_{0}^{2} v_{0}^{2}}{4} W_{\mu}^{+} W^{-\mu}+Z^{\chi} \frac{g_{0}^{2} v_{0}^{2}}{8 c_{0}^{2}} Z_{\mu} Z^{\mu}+\ldots
$$

It is clear that $Z^{\phi}$ denotes the self energy correction of the charged Goldstone boson $\phi^{ \pm}$, and $Z^{\chi}$ denotes the self energy correction of the neutral Goldstone boson $\phi^{3}$. Since $W_{\mu}^{ \pm}$and $Z_{\mu}$ do not get wavefunction correction in powers of $m_{t}$, therefore the gauge boson masses are

$$
\begin{aligned}
& M_{W}^{2}=Z^{\phi} \frac{g_{0}^{2} v_{0}^{2}}{4}=Z^{\phi} M_{W 0}^{2} \\
& M_{Z}^{2}=Z^{\chi} \frac{g_{0}^{2} v_{0}^{2}}{4 c_{0}^{2}}=Z^{\chi} M_{Z_{0}}^{2} .
\end{aligned}
$$

In summary, all the loop corrections in powers of $m_{t}$ to low energy data can be organized in the sum of $\mathcal{L}_{\text {eff }}$ [in Eq. (39)] and $\mathcal{L}_{\text {eff }}^{B}[$ in Eq. (40)]. Comparing them to the bare Lagrangian $\mathcal{L}_{0}$ in Eq. (29), we find that in the limit of taking $g \rightarrow 0$ the chiral Lagrangian $\mathcal{L}_{0}$ behaves as a renormalizable theory although in general a chiral Lagrangian is nonrenormalizable. In other words, no higher dimensional operators (counterterms) are needed to renormalize the theory in this limit. The same feature was also found in another application of a chiral Lagrangian with $1 / N$ expansion 23.

\subsection{Renormalization}

Now we are ready to consider the large $m_{t}$ corrections to low energy data. We choose our renormalization scheme to be the $\alpha, G_{F}$, and $M_{Z}$ scheme. With

$$
g_{0}^{2}=\frac{4 \pi \alpha_{0}}{s_{0}^{2}}
$$

and

$$
s_{0}^{2} c_{0}^{2}=\frac{\pi \alpha_{0}}{\sqrt{2} G_{F 0} M_{Z 0}^{2}},
$$

or,

$$
s_{0}^{2}=\frac{1}{2}\left[1-\left(1-\frac{4 \pi \alpha_{0}}{\sqrt{2} G_{F 0} M_{Z_{0}}^{2}}\right)^{1 / 2}\right] .
$$

Define the counterterms as

$$
\begin{aligned}
\alpha & =\alpha_{0}+\delta \alpha, \\
G_{F} & =G_{F 0}+\delta G_{F}, \\
M_{Z}^{2} & =M_{Z 0}^{2}+\delta M_{Z}^{2},
\end{aligned}
$$


and

$$
\begin{aligned}
& s^{2}=s_{0}^{2}+\delta s^{2}=s_{0}^{2}-\delta c^{2}, \\
& c^{2}=c_{0}^{2}+\delta c^{2},
\end{aligned}
$$

then

$$
s^{2} c^{2}+\left(c^{2}-s^{2}\right) \delta c^{2}=\frac{\pi \alpha}{\sqrt{2} G_{F} M_{Z}^{2}}\left(1-\frac{\delta \alpha}{\alpha}+\frac{\delta G_{F}}{G_{F}}+\frac{\delta M_{Z}^{2}}{M_{Z}^{2}}\right) .
$$

As shown in the above equation, even after the counterterms $\delta \alpha, \delta G_{F}$, and $\delta M_{Z}^{2}$ are fixed by data [e.g., the electron (g-2), muon lifetime, and the mass of the $Z$ boson], we still have the freedom to choose $\delta c^{2}$ by using a different definition of the renormalized quantity $s^{2} c^{2}$. In our case we would choose the definition of the renormalized $s^{2}$ such that there will be no large top quark mass dependence (in powers of $m_{t}$ ) in the counterterm $\delta c^{2}$. We shall show later that for this purpose our renormalized $s^{2}$ satisfies

$$
s^{2} c^{2}=\frac{\pi \alpha}{\sqrt{2} G_{F} M_{Z}^{2} \rho},
$$

where $\rho$ is defined from the partial width of $Z$ into lepton pairs, cf. Eq. (67). With this choice of $s^{2}$ and the definition of the renormalized weak coupling

$$
g^{2}=\frac{4 \pi \alpha}{s^{2}}
$$

one can easily show that the counterterm $\delta g^{2}\left(=g^{2}-g_{0}^{2}\right)$ does not contain large $m_{t}$ dependence. (Obviously, $\delta \alpha$ will not have contributions purely in powers of $m_{t}$.) Namely, in this renormalization scheme, $\alpha, g$, and $s^{2}$ do not get renormalized after ignoring all the contributions of the order $g$. Hence, all the bare couplings $g_{0}, g_{0}^{\prime}$, and $s_{0}^{2}$ in the effective Lagrangians $\mathcal{L}_{\text {eff }}$ and $\mathcal{L}_{\text {eff }}^{B}$ do not get corrected when considering the contributions which do not vanish in the limit of $g \rightarrow 0$. The only non-vanishing counterterm needs to be considered in Eq. (40) is $\delta v^{2}\left(=v^{2}-v_{0}^{2}\right)$. From Eq. (42) and $M_{W}=g v / 2$, we find

$$
Z^{\phi} v_{0}^{2}=v^{2}
$$

\footnotetext{
${ }^{3}$ If we define $s^{\prime 2} c^{\prime 2}=\frac{\pi \alpha}{\sqrt{2} G_{F} M_{Z}^{2}}$, then $s^{2}=s^{2}\left(1+\Delta k^{\prime}\right)$ with $\Delta k^{\prime}=-\frac{c^{\prime 2} \delta \rho}{c^{\prime 2}-s^{\prime 2}}$, and the counterterm of $s^{2}$ will contain contributions in powers of $m_{t}$.
} 
because neither $g$ nor $\mathcal{W}^{ \pm}$(or $W^{ \pm}$) gets renormalized. Thus,

$$
G_{F 0}=\frac{1}{\sqrt{2} v_{0}^{2}}=Z^{\phi} \frac{1}{\sqrt{2} v^{2}}=Z^{\phi} G_{F}
$$

Consequently,

$$
\frac{g_{0}^{2}}{c_{0}^{2}}=\frac{8 G_{F 0} M_{Z 0}^{2}}{\sqrt{2}}=\frac{8 G_{F} M_{Z}^{2}}{\sqrt{2}} \frac{Z^{\phi}}{Z^{\chi}}
$$

and the effective $Z-b-b$ coupling is

$$
-\frac{g_{0}}{2 c_{0}} \gamma^{\mu}\left[\left(\frac{Z_{v}^{L}}{Z_{b}^{L}}-\frac{2 s_{0}^{2}}{3}\right) P_{L}-\frac{2 s_{0}^{2}}{3} P_{R}\right]=-\sqrt{\frac{G_{F} M_{Z}^{2}}{2 \sqrt{2}} \frac{Z^{\phi}}{Z^{\chi}}} \gamma^{\mu}\left[\left(\frac{Z_{v}^{L}}{Z_{b}^{L}}-\frac{4 s^{2}}{3}\right)-\frac{Z_{v}^{L}}{Z_{b}^{L}} \gamma_{5}\right]
$$

where $P_{L, R}=\left(1 \mp \gamma_{5}\right) / 2$.

\subsection{Low Energy Observables}

In general, all the radiative corrections to low energy data can be categorized in a model independent way into four parameters: $S, T, U$ [24], and $R_{b}$ [25]; or equivalently, $\epsilon_{1}, \epsilon_{2}, \epsilon_{3}$, and $\epsilon_{b}$ [26]. The relations between these two sets of parameters are, to the order of interest,

$$
\begin{aligned}
S & =\frac{4 s^{2}}{\alpha\left(M_{Z}^{2}\right)} \epsilon_{3}, \\
T & =\frac{1}{\alpha\left(M_{Z}^{2}\right)} \epsilon_{1}, \\
U & =-\frac{4 s^{2}}{\alpha\left(M_{Z}^{2}\right)} \epsilon_{2},
\end{aligned}
$$

and both $R_{b}\left(=\Gamma_{b} / \Gamma_{h}\right)$ and $\epsilon_{b}$ measure the effects of new physics in the partial decay width $\left(\Gamma_{b}\right)$ of $Z \rightarrow b \bar{b}$. ( $\Gamma_{h}$ is the hadronic width of $Z$.)

These parameters can be derived from four basic measured observables, such as $\Gamma_{\mu}$ (the partial decay width of $Z$ into a $\mu$ pair), $A_{F B}^{\mu}$ (the forward-backward asymmetry at the $Z$ peak for the $\mu$ lepton), $M_{W} / M_{Z}$ (the ratio of $W^{ \pm}$and $Z$ masses), and $\Gamma_{b}$ (the

partial decay width of $Z$ into a $b \bar{b}$ pair). The expressions of these observables in terms of $\epsilon$ 's can be found in Ref. [26].

In this paper we only give the relevant terms in $\epsilon$ 's that might contain the leading effects in powers of $m_{t}$ from new physics. Denote the vacuum polarization for the 
$W^{1}, W^{2}, W^{3}$, and $B$ gauge bosons as

$$
\Pi_{\mu \nu}^{i j}(q)=-i g_{\mu \nu}\left[A^{i j}(0)+q^{2} F^{i j}\left(q^{2}\right)\right]+q_{\mu} q_{\nu} \text { terms }
$$

where $i, j=1,2,3,0$ for $W^{1}, W^{2}, W^{3}$ and $B$, respectively. Then,

$$
\begin{gathered}
\epsilon_{1}=e_{1}-e_{5}, \\
\epsilon_{2}=e_{2}-c^{2} e_{5}, \\
\epsilon_{3}=e_{3}-c^{2} e_{5}, \\
\epsilon_{b}=e_{b},
\end{gathered}
$$

where

$$
\begin{gathered}
e_{1}=\frac{A^{33}(0)-A^{11}(0)}{M_{W}^{2}}, \\
e_{2}=F^{11}\left(M_{W}^{2}\right)-F^{33}\left(M_{Z}^{2}\right), \\
e_{3}=\frac{c}{s} F^{30}\left(M_{Z}^{2}\right) \\
e_{5}=M_{Z}^{2} \frac{d F^{Z Z}}{d q^{2}}\left(M_{Z}^{2}\right),
\end{gathered}
$$

and $e_{b}$ is defined through the Glashow-Iliopoulos-Maiani- (GIM-) violating $Z \rightarrow b \bar{b}$ vertex

$$
V_{\mu}^{\mathrm{GIM}}(Z \rightarrow b \bar{b})=-\frac{g}{2 c} e_{b} \gamma_{\mu} \frac{1-\gamma_{5}}{2} .
$$

Both $\epsilon_{1}$ and $\epsilon_{b}$ gain corrections in powers of $m_{t}$ [11], and are sensitive to new physics coming through the top quark. On the contrary, $\epsilon_{2}$ and $\epsilon_{3}$ do not play any significant role in our analysis because their dependence on the top mass is only logarithmic. Hence,

$$
\begin{aligned}
\epsilon_{1} & =\delta \rho+\text { corrections of the order } g, \\
\epsilon_{b} & =\tau+\text { corrections of the order } g, \\
\epsilon_{2} & =\text { corrections of the order } g, \\
\epsilon_{3} & =\text { corrections of the order } g,
\end{aligned}
$$


where $\delta \rho=\rho-1$. The parameters $\rho$ and $\tau$ are defined by

$$
\begin{aligned}
\Gamma_{\mu} & \equiv \Gamma\left(Z \rightarrow \mu^{+} \mu^{-}\right)=\rho \frac{G_{F} M_{Z}^{3}}{6 \pi \sqrt{2}}\left(g_{\mu_{V}}^{2}+g_{\mu_{A}}^{2}\right) \\
\Gamma_{b} & \equiv \Gamma(Z \rightarrow \bar{b} b)=\rho \frac{G_{F} M_{Z}^{3}}{2 \pi \sqrt{2}}\left(g_{b V}^{2}+g_{b A}^{2}\right)
\end{aligned}
$$

where

$$
\begin{aligned}
g_{\mu_{V}} & =-\frac{1}{2}\left(1-4 s^{2}\right), \quad g_{\mu_{A}}=-\frac{1}{2} \\
g_{b V} & =-\frac{1}{2}\left(1-\frac{4}{3} s^{2}+\tau\right), \quad g_{b A}=-\frac{1}{2}(1+\tau) .
\end{aligned}
$$

Hence, comparing to Eq. (54) we conclude

$$
\begin{aligned}
\delta \rho & =\frac{Z^{\phi}}{Z \chi}-1 \\
\tau & =\frac{Z_{v}^{L}}{Z_{b}^{L}}-1 .
\end{aligned}
$$

\subsection{One Loop Corrections in the SM}

The SM, being a linearly realized $\mathrm{SU}(2)_{L} \times \mathrm{U}(1)_{Y}$ gauge theory, can be formulated as a chiral Lagrangian after nonlinearly transforming the fields [11]. Applying the previous formalism, we calculate the one-loop corrections of order $m_{t}^{2}$ to $\rho$ and $\tau$ for the SM by taking $\kappa_{L}^{\mathrm{NC}}=\kappa_{R}^{\mathrm{NC}}=\kappa_{L}^{\mathrm{CC}}=\kappa_{R}^{\mathrm{CC}}=0$ in Eq. (29). These loop corrections can be summarized by the coefficient functions $Z^{\chi}, Z^{\phi}, Z_{b}^{L}$, and $Z_{v}^{L}$ which are calculated from the Feynman diagrams shown in Figs. 1(a), 1(b), 1(c), and the sum of 1(d) and $1(\mathrm{e})$, respectively. We find

$$
\begin{aligned}
& Z^{\chi}=1+\frac{6 m_{t}^{2}}{16 \pi^{2} v^{2}}\left(\Delta-\ln m_{t}^{2}\right) \\
& Z^{\phi}=1+\frac{6 m_{t}^{2}}{16 \pi^{2} v^{2}}\left(\Delta+\frac{1}{2}-\ln m_{t}^{2}\right), \\
& Z_{b}^{L}=1+\frac{3 m_{t}^{2}}{16 \pi^{2} v^{2}}\left(-\Delta+\ln m_{t}^{2}-\frac{5}{6}\right), \\
& Z_{v}^{L}=1+\frac{3 m_{t}^{2}}{16 \pi^{2} v^{2}}\left(-\Delta+\ln m_{t}^{2}-\frac{3}{2}\right)
\end{aligned}
$$

We note that Fig. 1(e) arises from the nonlinear realization of the gauge symmetry

in the chiral Lagrangian approach. Substituting the above results into Eq. (69), we 
obtain

$$
\begin{aligned}
\delta \rho & =\frac{3 G_{F} m_{t}^{2}}{8 \sqrt{2} \pi^{2}}, \\
\tau & =-\frac{G_{F} m_{t}^{2}}{4 \sqrt{2} \pi^{2}},
\end{aligned}
$$

which are the established results 15.

\section{Constraining the top quark couplings to the EW gauge bosons}

In Ref. [11] we calculated the one-loop corrections (of order $m_{t}^{2} \ln \Lambda^{2}$ ) to $\rho$ and $\tau$ due to the nonstandard couplings of the top quark to the EW gauge bosons. The set of Feynman diagrams we considered contained external massive gauge bosons lines. In this paper we show how to reproduce those results by considering a set of Feynman diagrams which contains only the pure Goldstone bosons, the top quark, and the bottom quark lines, as described in Sec. 2 .

Non-renormalizability of the effective Lagrangian presents a major problem on how to find a scheme to handle both the divergent and the finite pieces in loop calculations [27]. Such a problem arises because the underlying theory is not yet known, so it is not possible to apply the exact matching conditions to find the correct scheme to be used in the effective Lagrangian [28]. One approach is to associate the divergent piece in loop calculations with a physical cutoff $\Lambda$, the upper scale at which the effective Lagrangian is valid [19]. In the chiral Lagrangian approach this cutoff $\Lambda$ is taken to be $4 \pi v \sim 3 \mathrm{TeV}$ 28. . For the finite piece no completely satisfactory approach is available [27.

To perform loop calculations using the chiral Lagrangian, one should arrange the corrections in powers of $1 / 4 \pi v$ and include all the Feynman diagrams up to the desired order. Fig. 1 contains all the Feynman diagrams needed for our study. We calculate the leading contribution to $\rho$ and $\tau$ due to the new interaction terms in the chiral

\footnotetext{
${ }^{4}$ This scale, $4 \pi v \sim 3 \mathrm{TeV}$, is only meant to indicate the typical cutoff scale. It is equally probable to have, say, $\Lambda=1 \mathrm{TeV}$.
} 
Lagrangian using the dimensional regularization scheme and taking the bottom quark mass to be zero. At the end of the calculation, we replace the divergent piece $1 / \epsilon$ by $\ln \left(\Lambda^{2} / m_{t}^{2}\right)$ for $\epsilon=(4-n) / 2$, where $n$ is the space-time dimension. Effectively, we have assumed that the underlying full theory is renormalizable. The cutoff scale $\Lambda$ serves as the infrared cutoff of the operators in the effective Lagrangian. Due to the renormalizability of the full theory, from renormalization group analysis, we conclude that the same cutoff $\Lambda$ should also serve as the ultraviolet cutoff of the effective Lagrangian in calculating Wilson coefficients. Hence, in the dimensional regularization scheme, $1 / \epsilon$ is replaced by $\ln \left(\Lambda^{2} / \mu^{2}\right)$. Furthermore, the renormalization scale $\mu$ is set to be $m_{t}$, the heaviest mass scale in the effective Lagrangian of interest. Since we are mainly interested in new physics associated with the top quark couplings to gauge bosons, we shall restrict ourselves to the leading contribution enhanced by the top quark mass, i.e., of the order of $\left(m_{t}^{2} \ln \Lambda^{2}\right)$.

Inserting these nonstandard couplings in loop diagrams and keeping only the linear terms in $\kappa$ 's, we find

$$
\begin{aligned}
Z^{\chi} & =1+\frac{6 m_{t}^{2}}{16 \pi^{2} v^{2}}\left(2 \kappa_{L}^{\mathrm{NC}}-2 \kappa_{R}^{\mathrm{NC}}\right) \ln \frac{\Lambda^{2}}{m_{t}^{2}}, \\
Z^{\phi} & =1+\frac{12 m_{t}^{2}}{16 \pi^{2} v^{2}} \kappa_{L}^{\mathrm{CC}} \ln \frac{\Lambda^{2}}{m_{t}^{2}}, \\
Z_{b}^{L} & =1-\frac{6 m_{t}^{2}}{16 \pi^{2} v^{2}} \kappa_{L}^{\mathrm{CC}} \ln \frac{\Lambda^{2}}{m_{t}^{2}}, \\
Z_{v}^{L} & =1-\frac{m_{t}^{2}}{16 \pi^{2} v^{2}}\left(6 \kappa_{L}^{\mathrm{CC}}-4 \kappa_{L}^{\mathrm{NC}}+\kappa_{R}^{\mathrm{NC}}\right) \ln \frac{\Lambda^{2}}{m_{t}^{2}} .
\end{aligned}
$$

Thus the nonstandard contributions to $\rho$ and $\tau$ are

$$
\begin{aligned}
\delta \rho & =\frac{3 G_{F} m_{t}^{2}}{2 \sqrt{2} \pi^{2}}\left(\kappa_{L}^{\mathrm{CC}}-\kappa_{L}^{\mathrm{NC}}+\kappa_{R}^{\mathrm{NC}}\right) \ln \frac{\Lambda^{2}}{m_{t}^{2}}, \\
\tau & =\frac{G_{F} m_{t}^{2}}{2 \sqrt{2} \pi^{2}}\left(-\frac{1}{4} \kappa_{R}^{\mathrm{NC}}+\kappa_{L}^{\mathrm{NC}}\right) \ln \frac{\Lambda^{2}}{m_{t}^{2}},
\end{aligned}
$$

which agree with our previous results obtained in Ref. [11].

In Ref. [29] a similar calculation for $\tau$ was performed and the author claimed to get a different result from ours. However, the author included only the vertex corrections to calculate the physical quantity $\tau$, which according to our systematic discussion in 
the previous section is not complete because the wavefunction corrections to the $b$ quark must be included.

Based upon the new LEP measurements [13, a global analysis indicates a SM top quark mass to be [10]

$$
m_{t}=165 \pm 12 \mathrm{GeV} \text { for } m_{H}=300 \mathrm{GeV}
$$

If the SLC measurement is included with LEP measurements, then

$$
m_{t}=174 \pm 11 \mathrm{GeV} \text { for } m_{H}=300 \mathrm{GeV}
$$

Using the new LEP and SLC results we shall update the constraints on the nonstandard couplings of the top quark to the EW gauge bosons. This can be done by comparing the new experimental values for $\delta \rho$ and $\tau$ with that predicted by the SM and the nonstandard contributions combined. In the limit of ignoring the contributions of the order $g$, the observables $\Gamma_{\mu}, A_{F B}^{\mu}, M_{W} / M_{Z}$, and $\Gamma_{b}$ can all be expressed in terms of the two quantities $\delta \rho$ and $\tau$. In addition to Eq. (67), we find

$$
A_{F B}^{\mu}=\frac{3 g_{\mu_{V}}^{2} g_{\mu_{A}}^{2}}{\left(g_{\mu_{V}}^{2}+g_{\mu_{A}}^{2}\right)^{2}}
$$

and

$$
\frac{M_{W}^{2}}{M_{Z}^{2}}=\rho c^{2} .
$$

Using the minimum set of observables $\left(\Gamma_{\mu}, A_{F B}^{\mu}, M_{W} / M_{Z}\right.$, and $\left.\Gamma_{b}\right)$, we constrain the allowed space of $\kappa$ 's in a model independent way, i.e., without specifying the explicit dynamics for generating these nonstandard effects. One can also enlarge the set of observables used in the analysis by including all the LEP data and the SLC measurement of the left-right cross section asymmetry $A_{L R}$ in $Z$ production with a longitudinally polarized electron beam, where [26]

$$
A_{L R}=\frac{2 x}{1+x^{2}}
$$

\footnotetext{
${ }^{5}$ In terms of the quantity $\Delta r_{w}$ defined in Ref. [26], $\frac{M_{W}^{2}}{M_{Z}^{2}}\left(1-\frac{M_{W}^{2}}{M_{Z}^{2}}\right)=\frac{\pi \alpha\left(M_{Z}^{2}\right)}{\sqrt{2} G_{F} M_{Z}^{2}\left(1-\Delta r_{w}\right)}$. For corrections in powers of $m_{t}, s^{2} \Delta r_{w}=-c^{2} \delta \rho$.
} 
for

$$
x=\frac{g_{e V}}{g_{e A}}=1-4 s^{2} .
$$

Following the same analyses carried out in Ref. [11], we include both the SM and the nonstandard contributions to low energy data. The SM contributions to $\delta \rho$ and $\tau$ were given in Ref. [26] for various top quark and Higgs boson masses. Our conclusions are however not sensitive to the Higgs boson mass [11].

Choosing $m_{t}=175 \mathrm{GeV}$ and $m_{H}=100 \mathrm{GeV}$, we span the parameter space defined by $-1.0 \leq \kappa_{L}^{\mathrm{NC}} \leq 1.0,-1.0 \leq \kappa_{R}^{\mathrm{NC}} \leq 1.0$, and $-1.0 \leq \kappa_{L}^{\mathrm{CC}} \leq 1.0$, and compare with the values

$$
\begin{gathered}
\delta \rho=(3.5 \pm 1.8) \times 10^{-3} \\
\tau=(0.9 \pm 4.2) \times 10^{-3}
\end{gathered}
$$

from a global fit [10] using all the new LEP and SLC data. For reference, we list here some of the relevant data, taken from [10],

$$
\begin{aligned}
\alpha^{-1}\left(M_{Z}^{2}\right) & =128.87 \pm 0.12 \\
G_{F} & =1.16637(2) \times 10^{-5} \mathrm{GeV}^{-2} \\
M_{Z} & =91.1899 \pm 0.0044 \mathrm{GeV} \\
M_{W} / M_{Z} & =0.8814 \pm 0.0021 \\
\Gamma_{\ell} & =83.98 \pm 0.18 \mathrm{MeV} \\
\Gamma_{b} & =385.9 \pm 3.4 \mathrm{MeV} \\
A_{F B}^{\ell} & =0.0170 \pm 0.0016 \\
A_{F B}^{b} & =0.0970 \pm 0.0045 \\
A_{L R}(\mathrm{SLC}) & =0.1668 \pm 0.079
\end{aligned}
$$

We find that within $2 \sigma$ the allowed region of these three parameters exhibits the same features as that obtained using the old set of data (see Ref. [11]). These features can be deduced from the two-dimensional projections of the allowed parameter space, as shown in Figs. 2, 3, and 4. They are briefly summarized as follows:

\footnotetext{
${ }^{6} \epsilon_{1}=\delta \rho, \epsilon_{b}=\tau, \epsilon_{2}=(-9.2 \pm 5.1) \times 10^{-3}$, and $\epsilon_{3}=(3.8 \pm 1.9) \times 10^{-3}$.
} 
(1) As a function of the top quark mass, the allowed parameter space shrinks as the top quark mass increases.

(2) Data do not exclude possible new physics coming through the top quark couplings to the EW gauge bosons. As shown in Fig. $2, \kappa_{L}^{\mathrm{CC}}$ and $\kappa_{R}^{\mathrm{NC}}$ are not yet constrained by the current data. Furthermore, no conclusion can be drawn about $\kappa_{R}^{\mathrm{CC}}$ because $\kappa_{R}^{\mathrm{CC}}$ does not contribute to the LEP or the SLC observables in the limit of taking $m_{b}=0$.

(3) $\kappa_{L}^{\mathrm{NC}}$ is almost constrained. New physics prefers positive $\kappa_{L}^{\mathrm{NC}}$, see Figs. 3 and 4 . For example, $\kappa_{L}^{\mathrm{NC}}$ is constrained within $(-0.3$ to 0.5$)$ for a $175 \mathrm{GeV}$ top quark.

(4) New physics prefers $\kappa_{L}^{\mathrm{CC}} \approx-\kappa_{R}^{\mathrm{NC}}$. This is clearly shown in Fig. 2.

As compared with the old set of data from LEP and SLC, new data tighten the allowed region of the nonstandard parameters $\kappa$ 's by no more than a factor of two. This difference is due to the slightly smaller errors on the new measurements as compared with the old ones. The largest impact of these new data on our results comes from the more precise measurement of $\Gamma_{b}$ which turns out to be about $2 \sigma$ higher than the SM prediction and implies a lighter top quark. For a much heavier top quark, new physics must come in because all the $\kappa$ 's cannot simultaneously vanish. If the large discrepancy between LEP and SLC data persists, then our model of having nonstandard top quark couplings to the gauge bosons is one of the candidates that can accommodate such a difference.

If we restrict ourselves to the minimum set of observables, which give [10]

$$
\begin{gathered}
\delta \rho=(4.8 \pm 2.2) \times 10^{-3} \\
\tau=(5.0 \pm 4.8) \times 10^{-3}
\end{gathered}
$$

we reach almost the same conclusion. The main difference is that $\kappa_{L}^{\mathrm{NC}}$ shifts slightly to the right, due to the fact that the central value of $\tau$ in this case is larger than its global fit value. 
In Ref. [1] we discussed an effective model incorporated with an additional approximate custodial symmetry (responsible for $\rho=1$ at the tree-level), and concluded that $\kappa_{L}^{\mathrm{NC}}=2 \kappa_{L}^{\mathrm{CC}}$ as long as the tree-level vertex $b-b-Z$ is not modified. From Eq. (73), we find for this model

$$
\delta \rho=\frac{3 G_{F} m_{t}^{2}}{2 \sqrt{2} \pi^{2}}\left(-\frac{1}{2} \kappa_{L}^{\mathrm{NC}}+\kappa_{R}^{\mathrm{NC}}\right) \ln \frac{\Lambda^{2}}{m_{t}^{2}}
$$

and

$$
\tau=\frac{G_{F} m_{t}^{2}}{2 \sqrt{2} \pi^{2}}\left(-\frac{1}{4} \kappa_{R}^{\mathrm{NC}}+\kappa_{L}^{\mathrm{NC}}\right) \ln \frac{\Lambda^{2}}{m_{t}^{2}}
$$

Using this effective model, we span the plane defined by $\kappa_{L}^{\mathrm{NC}}$ and $\kappa_{R}^{\mathrm{NC}}$ for top quark mass $150 \mathrm{GeV}$ and $175 \mathrm{GeV}$, respectively. Figs. 5 and 6 show the allowed range for those parameters within $2 \sigma$. As a general feature one observes that the allowed range forms a narrow area aligned close to the line $\kappa_{L}^{\mathrm{NC}}=2 \kappa_{R}^{\mathrm{NC}}$. For $m_{t}=150 \mathrm{GeV}$ $(175 \mathrm{GeV})$ we see that $-0.05 \leq \kappa_{L}^{\mathrm{NC}} \leq 0.3\left(0.0 \leq \kappa_{L}^{\mathrm{NC}} \leq 0.25\right)$. As the top quark mass increases this range shrinks and moves downward to the right, away from the origin $\left(\kappa_{L}^{\mathrm{NC}}, \kappa_{R}^{\mathrm{NC}}\right)=(0,0)$, although positive $\kappa$ 's remain preferred. The reason for this behavior is simply due to the fact that as $m_{t}$ increases, the SM value for $\rho(\tau)$ increases in the positive (negative) direction. To summarize this behaviour, we show, respectively, in Figs. 7 and 8 the allowed ranges for $\kappa_{L}^{\mathrm{CC}}$ and $\left(\kappa_{L}^{\mathrm{NC}}-2 \kappa_{R}^{\mathrm{NC}}\right)$ as a function of $m_{t}$. An interesting point to mention is that in the global fit analysis the SM ceases to be a solution for $m_{t} \geq 200 \mathrm{GeV}$. However, with new physics effects, e.g., $\kappa_{L}^{\mathrm{CC}} \neq 0$, $m_{t}$ can be as large as $300 \mathrm{GeV}$.

In this analysis we concentrated on physics at the $Z$ resonance, i.e., at LEP and SLC. Other low energy observables may as well be used to constrain the nonstandard couplings of the top quark to the gauge bosons. In Ref. [30] a constraint on the righthanded charged current, $\kappa_{R}^{\mathrm{CC}}$, was set using the CLEO measurement of $b \rightarrow s \gamma$. The authors concluded that $\kappa_{R}^{\mathrm{CC}}$ is well constrained to within a few percent from its SM value $\left(\kappa_{R}^{\mathrm{CC}}=0\right)$. This provides a complementary information to our result because LEP and SLC data are not sensitive to $\kappa_{R}^{\mathrm{CC}}$ as compared to $\kappa_{L}^{\mathrm{CC}}, \kappa_{L}^{\mathrm{NC}}$, and $\kappa_{R}^{\mathrm{NC}}$. 


\section{Conclusions}

Because the top quark is heavy (close to the symmetry-breaking scale) it will be more sensitive than the other light fermions to new physics from the SSB sector. Concentrating on effects, to low energy data, directly related to the SSB sector, we took

the chiral Lagrangian approach to examine whether the nonstandard couplings, $\kappa$ 's, of the top quark to the gauge bosons $\left(W^{ \pm}\right.$and $Z$ ) were already strongly constrained by the old (1993) data from LEP and SLC [11]. Surprisingly, we found that to the order of $\left(m_{t}^{2} \ln \Lambda^{2}\right)$ only the left-handed neutral current, $\kappa_{L}^{\mathrm{NC}}$, was somewhat constrained by the precision low energy data, although data did impose some correlations among $\kappa_{L}^{\mathrm{NC}}, \kappa_{R}^{\mathrm{NC}}$, and $\kappa_{L}^{\mathrm{CC}}$. Since $\kappa_{R}^{\mathrm{CC}}$ does not contribute to the LEP or the SLC observables in the limit $m_{b}=0, \kappa_{R}^{\mathrm{CC}}$ cannot be constrained by these data. However, it was shown in Ref. [30] that $\kappa_{R}^{\mathrm{CC}}$ was already constrained by the complementary process $b \rightarrow s \gamma$.

In Ref. [11] we obtained our results by considering a set of Feynman diagrams, derived form the nonlinear chiral Lagrangian, whose external lines were the massive gauge boson lines. The leading corrections (in power of $m_{t}$ ) to the low energy observables were found not to vanish in the limit of vanishing $g$ (the weak coupling) because they originate from strong couplings to the SSB sector, e.g., through large Yukawa coupling $g_{t}$. Therefore, our previous results should in principle be reproduced by considering an effective Lagrangian which involves only the scalar (the unphysical Goldstone bosons and probably the Higgs boson) and the top-bottom fermionic sectors. This was shown in Sec. 2. We discussed how to relate the two corresponding sets of Green's functions for the low energy observables of interest. We showed that by considering a completely different set of Green's functions (without involving any external gauge boson line) from that discussed in Ref. [11] we obtained exactly the same results. Our result for $\tau$ is different from that given in Ref. [29] where the wavefunction correction to the bottom quark was not included.

In Sec. 3 we used the new (1994) LEP and SLC data to constrain the nonstandard interactions of the top quark to the EW gauge bosons. As compared with the old (1993) data from LEP and SLC, the new data tighten the allowed region of the 
nonstandard parameters, $\kappa$ 's, by no more than a factor of two. This difference is mainly due to the more precise measurement of $\Gamma_{b}$ which turns out to be about $2 \sigma$ higher than the SM prediction and favors a lighter top quark. If the large discrepancy between LEP and SLC data persists, then our model of having nonstandard top quark couplings to the gauge bosons is one of the candidates that can accommodate such a difference. Positive values for $\kappa$ 's are preferred for the special model discussed in Ref. [11], where an approximate custodial symmetry is assumed.

\section{Acknowledgments}

We thank X. Zhang for drawing our attention to his work in Ref. [29]. We also thank W. Repko for a critical reading of the manuscript. This work was supported in part by NSF Grant No. PHY-9309902.

\section{References}

[1] H. Georgi, Nucl. Phys. B363, 301 (1991).

[2] T. Appelquist and G.-H. Wu, Phys. Rev. D48, 3241 (1993); and the references therein.

[3] M. Golden and L. Randall, Nucl. Phys. B362, 3 (1991);

R.D. Peccei and S. Peris, Phys. Rev. D44, 809 (1991);

A. Dobado et al., Phys. Lett. B255, 405 (1991);

M. Dugan and L. Randall, Phys. Lett. B264, 154 (1991).

[4] F. Feruglio, Int. J. Mod. Phys. A8, 4937 (1993); and the references therein.

[5] B. Holdom and J. Terning, Phys. Lett. 247B, 88 (1990).

[6] J. Wudka, Int. J. Mod. Phys. A9, 2301 (1994); and the references therein.

[7] S. Abachi et al., Phys. Rev. Lett. 72, 2138 (1994).

[8] F. Abe et al. The CDF Collaboration, Phys. Rev. D50, 2966 (1994). 
[9] The SLD Collaboration (K. Abe et al.), Phys. Rev. Lett. 73, 25 (1994).

[10] G. Altarelli, CERN-TH-7319, June 1994.

[11] Ehab Malkawi and C.-P. Yuan. Phys. Rev. D50, 4462 (1994).

[12] The LEP Collaborations ALEPH, DELPHI, L3, OPAL and The LEP Electroweak Working Group, CERN/PPE/93-157 (1993);

W. Hollik, in Proceedings of the XVI International Symposium on LeptonPhoton Interactions, Cornell University, Ithaca, N.Y., Aug. 10-15, 1993;

M. Swartz, in Proceedings of the XVI International Symposium on LeptonPhoton Interactions, Cornell University, Ithaca, N.Y., Aug. 10-15, 1993;

B. Mele, in XIV Encontro Nacional de Fisica de Campos e Particulas, Caxambu, Brazil, 29 Sept.-3 Oct., 1993;

J. Lefrancois, in Proceedings of the EPS Conference on High Energy Physics, Marseille, France, 1993.

[13] M. Koratsinos and S. de Jong, in Proceedings of the Rencontres de la Vallee d'Aoste, La Thuile, 1994 ;

P. Clarke, P. Siegrist and B. Pietrzyk, in Proceedings of the Rencontres de Moriond, Meribel, 1994.

[14] M. Woods, in Proceedings of the Rencontres de Moriond, Meribel, 1994.

[15] R. Barbieri et al., Nucl. Phys. B409, 105 (1993);

R. Barbieri et al., Nucl. Phys. B288, 95 (1992).

[16] S. Coleman, J. Wess and B. Zumino, Phys. Rev. D177, 2239 (1969);

C.G. Callan, S. Coleman, J. Wess and B. Zumino, Phys. Rev. D177, 2247 (1969).

[17] M. Chanowitz, M. Golden, and H. Georgi. Phys. Rev. D36, 1490 (1987).

[18] S. Weinberg, Physica 96A, 327 (1979).

[19] R.D. Peccei and X. Zhang, Nucl. Phys. B337, 269 (1990). 
[20] H. Georgi, Nucl. Phys. B363, 301 (1991).

[21] F. Feruglio, A. Masiero and L. Maiani, Nucl. Phys B387, 523 (1992).

[22] H. Georgi, Weak Interactions and Modern Particle Theory (The Benjamin/Cummings Publishing Company, 1984).

[23] S. Naculich and C.-P. Yuan, Phys. Rev. D48, 1097 (1993).

[24] M.E. peskin and T. Takeuchi, Phys. Rev. Lett. 65, 964 (1990); Phys. Rev. D46, 381 (1991);

D.C. Kennedy and P. Langacker, Phys. Rev. Lett. 65, 2967 (1990);

W. Marciano and J. Rosner, Phy. Rev. D44, 1591 (1991);

B. Holdem, Phys. Lett. B259, 329 (1991).

[25] J. Bernabeu, A. Pich and A. Santamaria, Phys. Lett. B200, 569 (1988);

W. Beenakker and W. Hollik, Z. Phys. C40, 141 (1988);

A.A. Akhundov, D.Yu. Bardin and T. Riemann, Nucl. Phys. B276, 1 (1988).

[26] G. Altarelli, R. Barbieri, and F. Caravaglios, Nucl. Phys. B405, 3 (1993);

G. Altarelli and R. Barbieri, Phys. Lett. B253, 161 (1990);

G. Altarelli, R. Barbieri and S. Jadach, Nucl. Phys. B369, 3 (1992).

[27] C.P. Burgess and D. London, Phys. Rev. D48, 4337 (1993);

M.B. Einhorn, UM-TH-93-12, Apr 1993.

[28] H. Georgi, Nucl. Phys. B361, 339 (1991).

[29] X. Zhang, Mod. Phys. Lett. A9, 1955 (1994).

[30] K. Fujikawa and A. Yamada, Phys. Rev. D49, 5890 (1994). 


\section{Figure Captions}

Fig. 1.

The relevant Feynman diagrams, which contribute to $\rho$ and $\tau$ to the order $O\left(m_{t}^{2} \ln \Lambda^{2}\right)$.

Fig. 2.

Two-dimensional projection in the plane of $\kappa_{R}^{\mathrm{NC}}$ and $\kappa_{L}^{\mathrm{CC}}$, for $m_{t}=175 \mathrm{GeV}$ and $m_{H}=100 \mathrm{GeV}$.

Fig. 3.

Two-dimensional projection in the plane of $\kappa_{L}^{\mathrm{NC}}$ and $\kappa_{R}^{\mathrm{NC}}$, for $m_{t}=175 \mathrm{GeV}$ and $m_{H}=100 \mathrm{GeV}$.

Fig. 4.

Two-dimensional projection in the plane of $\kappa_{L}^{\mathrm{NC}}$ and $\kappa_{L}^{\mathrm{CC}}$, for $m_{t}=175 \mathrm{GeV}$ and $m_{H}=100 \mathrm{GeV}$.

Fig. 5.

The allowed region of $\kappa_{L}^{\mathrm{NC}}$ and $\kappa_{R}^{\mathrm{NC}}\left(\kappa_{L}^{\mathrm{NC}}=2 \kappa_{L}^{\mathrm{CC}}\right)$, for $m_{t}=150 \mathrm{GeV}$ and $m_{H}=$ $100 \mathrm{GeV}$.

Fig. 6.

The allowed region of $\kappa_{L}^{\mathrm{NC}}$ and $\kappa_{R}^{\mathrm{NC}}\left(\kappa_{L}^{\mathrm{NC}}=2 \kappa_{L}^{\mathrm{CC}}\right)$, for $m_{t}=175 \mathrm{GeV}$ and $m_{H}=$ $100 \mathrm{GeV}$.

Fig. 7.

The allowed range of $\kappa_{L}^{\mathrm{CC}}$ as a function of the mass of the top quark. (Note that $\kappa_{L}^{\mathrm{NC}}=2 \kappa_{L}^{\mathrm{CC}}$.)

Fig. 8.

The allowed range of $\kappa_{L}^{\mathrm{NC}}-2 \kappa_{R}^{\mathrm{NC}}$ as a function of the mass of the top quark. (Note that $\kappa_{L}^{\mathrm{NC}}=2 \kappa_{L}^{\mathrm{CC}}$.) 
This figure "fig1-1.png" is available in "png" format from: http://arxiv.org/ps/hep-ph/9502225v2 
This figure "fig1-2.png" is available in "png" format from: http://arxiv.org/ps/hep-ph/9502225v2 
This figure "fig1-3.png" is available in "png" format from: http://arxiv.org/ps/hep-ph/9502225v2 
This figure "fig1-4.png" is available in "png" format from: http://arxiv.org/ps/hep-ph/9502225v2 
This figure "fig1-5.png" is available in "png" format from: http://arxiv.org/ps/hep-ph/9502225v2 
This figure "fig1-6.png" is available in "png" format from: http://arxiv.org/ps/hep-ph/9502225v2 
This figure "fig1-7.png" is available in "png" format from: http://arxiv.org/ps/hep-ph/9502225v2 
This figure "fig1-8.png" is available in "png" format from: http://arxiv.org/ps/hep-ph/9502225v2 